\title{
ESCALAS UTILIZADAS PARA PREVENIR ÚLCERAS POR PRESSÃO EM PACIENTES CRÍTICOS
}

\author{
Mariana Pinheiro Santos Enfermeira graduada pela Escola \\ Bahiana de Medicina e Saúde \\ Pública \\ Rebecca Costa Neves \\ Enfermeira graduada pela Escola \\ Bahiana de Medicina e Saúde \\ Pública \\ Carina Oliveira dos Santos \\ Fisioterapeuta MSc, docente da \\ Escola Bahiana de Medicina e \\ Saúde Pública e da Universidade \\ Estadual da Bahia
}

\begin{abstract}
Resumo
Pacientes críticos apresentam risco de desenvolver úlcera por pressão, representando um grupo prioritário para o estudo e identificação desse agravo. Para prevenir essas lesões se faz necessária a aplicação de instrumentos de avaliação específicos. O objetivo do estudo foi identificar, na literatura nacional, as escalas utilizadas para prevenir úlceras por pressão em pacientes críticos e o papel do enfermeiro. Trata-se de um estudo de revisão integrativa da literatura que utiliza como base a pesquisa bibliográfica do tipo descritiva e exploratória com caráter qualitativo. O rastreamento bibliográfico foi realizado entre os anos de 2004 a 2012 a partir das bases de dados científicas do LILACS e SciELO. Todos os periódicos estiveram disponibilizados na íntegra e na língua nacional. Os resultados mostraram que os pacientes críticos apresentam maior risco de desenvolver úlceras por pressão sendo fundamental que ações de prevenção sejam implantadas em unidades nas quais os pacientes estejam susceptíveis a esse agravo. A utilização de um instrumento de predição para o desenvolvimento de úlcera por pressão permite conhecer o risco individual de cada paciente para que se possa implementar ações de enfermagem preventivas. Conclui-se que a escala de Braden apresentou maior preditividade e sensibilidade, segundo a maioria dos autores em relação às escalas de Norton e Waterlow.
\end{abstract}

Palavras-chave: Úlcera por pressão; Medição de risco; Pacientes críticos; Escalas preditivas.

\section{SCALES USED TO PREVENT PRESSURE ULCERS IN CRITICAL PATIENTS}

\begin{abstract}
Critically ill patients at risk of developing pressure ulcers, represented a priority group for the study and identification of this condition. To prevent these injuries is necessary to apply specific assessment instruments. The aim of the study was to identify the national literature of the scales used to prevent pressure ulcers in critically ill patients and the role of the nurse. This is a study of integrative literature review using as a base a bibliographic research of a descriptive and exploratory qualitative character. The tracking literature review was conducted between 2004 to 2012 from the database of scientific LILACS and SciELO. All journals were available in full and in the national language. The results showed that critically ill patients are at increased risk of developing pressure ulcers is essential that preventive actions are implemented in units in which patients are susceptible to these diseases. The use of a prediction tool for the development of pressure ulcers allows to know the individual risk of each patient so that we can implement preventive nursing actions. We conclude that the Braden scale showed greater predictability and sensitivity, according to most authors in relation to Norton and Waterlow scales.
\end{abstract}

Keywords: Pressure ulcer; Risk measurement; Critical patients; Predictive scales. 


\title{
ESCALAS UTILIZADAS PARA PREVENIR LAS ÚLCERAS POR PRESIÓN EN PACIENTES EN ESTADO CRÍTICO
}

\begin{abstract}
Resumen
Los pacientes críticos presentan riesgo de desarrollar úlceras por presión, representando un grupo prioritario para el estudio e identificación de esta condición. Para evitar estas lesiones es necesario aplicar instrumentos de evaluación específicos. El objetivo del estudio fue identificar en la literatura nacional las escalas utilizadas para prevenir las úlceras por presión en pacientes en estado crítico y el papel del enfermero. Este es un estudio de revisión integradora de la literatura que utiliza como base la investigación bibliográfica descriptiva y exploratoria de carácter cualitativa. La revisión de la literatura se llevó a cabo de 2004 a 2012, utilizando como base de datos científicos LILACS y SciELO. Todos los periódicos estaban disponibles en su totalidad y en el idioma nacional. Los resultados mostraron que los pacientes críticamente enfermos presentan mayor riesgo de desarrollar úlceras por presión siendo esencial que acciones preventivas sean implementadas en las unidades en que los pacientes son susceptibles a esta enfermedad. El uso de una herramienta de predicción para el desarrollo de las úlceras por presión permite conocer el riesgo individual de cada paciente, de manera que se pueda poner en práctica las acciones de enfermería preventivas. Se concluye que la escala de Braden mostró una mayor previsibilidad y sensibilidad, según la mayoría de los autores, en relación a las escalas de Norton y Waterlow.
\end{abstract}

Palabras clave: Úlcera por presión; Medición de riesgo; Pacientes críticos; Escalas de predicción.

\section{INTRODUÇÃOO}

A úlcera por pressão (UP) é uma lesão localizada resultante da compressão, podendo estar associada à fricção ou cisalhamento do tecido mole entre uma proeminência óssea e uma superfície rígida, acometendo pele e/ou tecidos subjacentes. A lesão se instala quando ocorre uma pressão tecidual maior que a pressão de perfusão capilar, por um período de tempo maior que o necessário para o tecido se recuperar da isquemia originada. ${ }^{(1)}$

Os pacientes em estado crítico apresentam características peculiares em razão da gravidade das suas condições clínicas, pois requerem maior restrição ao leito, terapias complexas, procedimentos invasivos, maior tempo de permanência hospitalar e maior necessidade de manipulação, o que os faz mais susceptíveis a complicações e com maior probabilidade de desenvolver UP tornando-se grupo de risco. Além disso, esses pacientes podem apresentar integridade da pele prejudicada devido à má nutrição, utilização de drogas vasoativas e sedativas, desidratação, imobilidade, edema, idade avançada, incontinências, anemia, entre outros. ${ }^{(2)}$

A UP pode ser evitada com o conhecimento dos fatores associados ao seu processo de formação e para tanto é necessário que o profissional enfermeiro busque detectar, precocemente, o paciente com potencial de risco para esse tipo de lesão. ${ }^{(2)} \mathrm{Um}$ dos meios utilizados na prevenção da UP é a aplicação de escalas preditivas que auxiliam na implantação de medidas específicas preventivas, direcionando as intervenções de enfermagem. 
No intuito de proporcionar mais subsídios no sentido de aperfeiçoar e estender a habilidade clínica dos enfermeiros, diversos autores criaram escalas de análise de risco, dentre as quais, as mais citadas na literatura são a de Norton, Waterlow e Braden, aplicadas para prevenir e detectar UP, apresentando adequados índices de validade preditiva, sensibilidade e especificidade. A aplicabilidade deste instrumento de avaliação nas instituições hospitalares pode determinar a modificação no processo de assistência e redução na incidência de novos casos. $^{(3)}$

O maior entendimento sobre os instrumentos utilizados para avaliar o risco de desenvolver UP e sua aplicabilidade poderá contribuir para prevenir essas lesões em pacientes críticos, tornando a equipe de enfermagem capacitada para realizar avaliação adequada das condições da pele e a partir dela implementar cuidados, a fim de prevenir lesões e restaurar as já instaladas. Além disso, devem ser propostas novas estratégias para a prevenção - pois este é um grave problema da equipe multiprofissional associado à má qualidade da assistência e exige uma grande demanda de tempo e dinheiro para tratamento das lesões, sobretudo quando a prevenção recebe menos atenção - quando não existem programas específicos voltados para esse problema, acarretando prejuízos estruturais e estéticos aos pacientes, prolongando o tempo de internamento, além de ser uma via de grande potencial para o surgimento e disseminação de infecções hospitalares..$^{(2,4,5)}$

Uma estratificação regular e precoce baseada nos risco de desenvolver UP é fundamental para a adoção de medidas terapêuticas adequadas para a redução dos fatores predisponentes e otimização do estado geral do paciente. Para alcançar essa estratégia terapêutica se faz necessário a experiência do profissional de saúde e insumos. Dessa maneira, é notória a importância da realização de estudos que avaliem técnicas, instrumentos e produtos na prevenção de UP, passíveis de adaptação para os diversos cenários dos serviços de saúde. Neste quesito, é destacável o uso de métodos indiretos como as escalas de avaliação de risco, pois são eficazes, práticas e econômicas. ${ }^{(4,6,7,8,9)}$

A afinidade com o tema e as experiências práticas vividas durante os estágios proporcionaram o estímulo para o desenvolvimento desta pesquisa, pois mostraram a não utilização das escalas e a falta de conhecimento dos profissionais.

Portanto, o presente estudo tem como objetivo analisar as escalas utilizadas para prevenir UP em pacientes críticos e o papel do enfermeiro, tendo como objetivos específicos: comparar a aplicabilidade e especificidade das escalas; vantagens/desvantagens das escalas estudadas e o papel do enfermeiro na prevenção das úlceras por pressão. 


\section{METODOLOGIA}

Trata-se de uma revisão de literatura integrativa baseada na pesquisa bibliográfica formulada através de artigos publicados sobre os instrumentos utilizados para prevenir UP.

A revisão de literatura integrativa permite a incorporação das evidências na prática clínica, tendo como finalidade reunir e sintetizar resultados de pesquisa sobre um determinado tema em questão, de maneira sistemática e ordenada, contribuindo para o aprofundamento do conhecimento do tema investigado. ${ }^{(10)}$

Na delimitação do tema da pesquisa, foi realizado um estudo descritivo exploratório de caráter qualitativo, que, segundo Andrade, ${ }^{(11)}$ está voltado para registrar, analisar e interpretar os fatos de forma que o pesquisador não interfira nos mesmos, exigindo do autor uma delimitação precisa de técnicas, métodos e modelos que orientem na interpretação dos dados. O objetivo é conferir validade científica da pesquisa buscando trabalhos de natureza teórica capazes de proporcionar explicações a respeito do tema proposto, bem como de pesquisas que abordam o assunto.

Para tanto, foi realizado um levantamento bibliográfico informativo indexado nas bases de dados: Scientific Eletronic Library Online (SciELO) e Literatura Latino-Americana em Ciências da Saúde e do Caribe (LILACS) utilizando os seguintes descritores: úlcera por pressão, medição de risco, paciente crítico, escalas preditivas.

Os critérios de inclusão da referida pesquisa foram pautados no acesso aos artigos publicados em periódicos nacionais, na íntegra, que trouxessem abordagem plena e/ou parcial do objeto de estudo e que tenham sido publicados no período de 2004 a 2012.

Para a coleta de dados, realizamos a leitura interpretativa dos artigos sendo possível elencar o material, extrair dos textos temas de interesse nesta pesquisa e interpretá-los a partir do objetivo proposto.

A análise dos dados foi feita por meio da seleção dos artigos, categorização e apresentação em forma de tabela através do programa Microsoft Office Excel 2007. Essa etapa se apresentou como o início dos resultados e teve a finalidade de ordenar e sumarizar as informações contidas nos periódicos. Focou-se de maneira sistemática na região do estudo, nas escalas mais citadas e nos anos de publicação. Posteriormente foram identificadas de maneira interpretativa e descritiva as categorias do estudo.

A realização do estudo respeitou os trâmites legais de acordo com os aspectos éticos presentes no Código de Ética dos Profissionais de Enfermagem no que se refere ao ensino, 
pesquisa e produção técnico-científica. Em relação às responsabilidades e deveres, "respeitar os princípios da honestidade e fidedignidade, bem como os direitos autorais no processo de pesquisa, especialmente na divulgação dos seus resultados” (Art. 91 do Código).

\section{RESULTADOS E DISCUSSÃO}

Para apresentar os resultados, estes foram analisados e discutidos com base nos trabalhos que fazem referência ao objetivo do estudo, proporcionando uma discussão prévia dos resultados, sendo considerados como a primeira etapa dessa apresentação.

Foram encontrados 41 artigos de acordo com os descritores do estudo, porém, após uma leitura integral, foram selecionados apenas 16 conforme o critério de inclusão.

Inicialmente, os artigos foram catalogados em forma de quadro para melhor visualização dos conteúdos descritos por seus autores, descrevendo as regiões de estudo, as escalas mais citadas e os anos de publicação (Quadro 1).

Quadro 1 - Regiões, anos e escalas pesquisadas

\begin{tabular}{|c|c|}
\hline REGIÕES DE ESTUDO & Quantidade de artigos \\
\hline São Paulo & 10 \\
\hline Rio de Janeiro & 1 \\
\hline Rio Grande do Sul & 2 \\
\hline Pernambuco & 1 \\
\hline Minas Gerais & 1 \\
\hline Brasília & 1 \\
\hline $\begin{array}{c}\text { ANO DE } \\
\text { PUBLICAÇÃO }\end{array}$ & Quantidade de artigos \\
\hline 2012 & 1 \\
\hline 2011 & 4 \\
\hline 2010 & 3 \\
\hline 2009 & 1 \\
\hline 2008 & 1 \\
\hline 2007 & 2 \\
\hline 2006 & 1 \\
\hline 2005 & 1 \\
\hline
\end{tabular}

Revista Enfermagem Contemporânea. 2013 Ago;2(1):19-31. 


\begin{tabular}{c|c}
\hline $\mathbf{2 0 0 4}$ & 2 \\
\hline ESCALAS CITADAS & Quantidade de artigos \\
\hline Escala de Braden & $14 / 16$ \\
\hline Escala de Norton & $2 / 16$ \\
\hline Escala de Waterlow & $3 / 16$ \\
\hline
\end{tabular}

De acordo com o Quadro 1, o estado mais próspero em publicações foi São Paulo, local onde foram realizados dez artigos.

Outra análise do estudo foi o ano de publicação dos artigos, a partir da qual se percebeu que este tema, por ser relevante e indispensável na prática da assistência à saúde, apresentou publicações durante todo o período estudado (2004 a 2012), com maior destaque para o ano de 2011, que apresentou maior número de publicações: quatro.

Por último, foi avaliada a citação das escalas. Obteve-se que a escala mais citada foi a de Braden, estando presente em 14 dos 16 artigos selecionados.

Portanto, a partir do objeto de estudo - análise das escalas utilizadas para prevenir úlcera por pressão em pacientes críticos e o papel do enfermeiro -, emergiram as seguintes categorias: perfil do paciente predisposto, análise da aplicabilidade e especificidade das escalas e o papel do enfermeiro na aplicação das escalas. Foi realizada a interpretação da ideia de cada autor, comparando os dados e fatos, concordâncias e divergências, sem interferência pessoal, a fim de detectar homogeneidades e heterogeneidades entre os estudos, atingindo os objetivos propostos.

\section{Perfil do paciente predisposto}

Os pacientes que apresentam maior perfil de desenvolver UP são aqueles em estado crítico. São considerados pacientes críticos aqueles que apresentam condições clínicas graves, requerendo controles mais frequentes e rigorosos, associados a terapias de maior complexidade, representando um grupo prioritário para o estudo e identificação da ocorrência dessas lesões. ${ }^{(12)}$

Pacientes que possuem, com frequência, instabilidade hemodinâmica, suporte ventilatório, necessidade de sedação, uso de drogas vasoativas, alterações do nível de consciência e restrição de movimentos por períodos prolongados de tempo, tornam-se mais 
expostos aos procedimentos invasivos e com maior necessidade de manipulação, sendo fatores de risco para o desenvolvimento de UP. ${ }^{(7)}$

Segundo Rocha e Barros, ${ }^{(13)}$ estão predispostos ao desenvolvimento dessas lesões pacientes que possuem associação de fatores intrínsecos e extrínsecos. Os fatores intrínsecos referem-se ao estado nutricional, mobilidade, incontinência e condições clínicas, tais como: doenças malignas, neurológicas e anemia. Já os extrínsecos incluem: os efeitos das drogas, distribuição de peso, regime de tratamento e cuidados corporais.

Blanes et al. ${ }^{(12)}$ demonstrou, através de estudos com pacientes em uma instituição hospitalar, que dos 78 pacientes portadores de UP, 66,7\% tinham idade acima de 61 anos associada ao uso de terapias complexas, comprovando que idade avançada e condições clínicas graves são fatores desencadeantes para o surgimento dessas lesões. Sabe-se que a diminuição da elasticidade, da circulação, do nível de reposição celular, do processo cicatricial, bem como da sensibilidade periférica são processos inerentes ao avanço da idade, levando ao aumento do risco de trauma tegumentar.

Estudo feito por Cremasco et al. ${ }^{(2)}$ em uma Unidade de Terapia Intensiva constatou que a maior parte dos pacientes que desenvolveram UP estava sob ventilação mecânica, uso de sedativos, de vasopressores e cardiotônicos. Esse perfil de paciente possui uma redução das respostas reflexas e protetoras do organismo, limitando sua mobilidade física e percepção sensorial, contribuindo para o surgimento dessas lesões.

Nota-se que nos estudos revisados os autores deixaram evidente que o principal fator de risco para o desenvolvimento de UP é o fato de os pacientes se encontrarem em estado crítico. A partir dessa ótica é fundamental que ações de prevenção sejam implantadas em unidades nas quais os pacientes estejam susceptíveis a esses agravos.

\section{Análise da aplicabilidade e especificidade das escalas}

Na tentativa de diminuir os índices de prevalência de UP e melhorar a qualidade de vida dos pacientes, são utilizadas escalas de prevenção que identificam os pacientes de risco para que se possa agir sistematicamente evitando essas lesões. Existem mais de 40 escalas, porém as mais utilizadas são a de Norton, a de Waterlow e a de Braden, diferindo apenas na abrangência, complexidade e aplicabilidade. ${ }^{(9)}$ 
Quadro 1 - Escalas

\begin{tabular}{|c|c|c|}
\hline Escalas de Norton & Escalas de Waterlow & Escalas de Braden \\
\hline Subescalas & Subescalas & Subescalas \\
\hline $\begin{array}{l}\text { Condição física; } \\
\text { Nível de consciência; } \\
\text { Atividade; } \\
\text { Mobilidade; } \\
\text { Incontinência. }\end{array}$ & $\begin{array}{l}\text { Peso/altura (IMC); } \\
\text { Avaliação visual da pele; } \\
\text { Sexo/idade; } \\
\text { Continência, } \\
\text { Mobilidade; } \\
\text { Apetite; } \\
\text { Medicações. }\end{array}$ & $\begin{array}{l}\text { Sensorial; } \\
\text { Atividade; } \\
\text { Mobilidade; } \\
\text { Umidade; } \\
\text { Nutrição; } \\
\text { Fricção ou cisalhamento. }\end{array}$ \\
\hline $\begin{array}{l}\text { Sub escalas: } 1 \text { a } 4 \text {; } \\
\text { Total: } 20 \text { pontos; } \\
\text { Quanto menor o escore } \\
\text { maior risco de UP; } \\
\text { Pontuação < } 12 \text { maior o } \\
\text { risco. } \\
(6)\end{array}$ & $\begin{array}{l}\text { Quanto maior o escore, maior } \\
\text { risco de UP: } \\
\text { Em risco (escore de } 10 \text { a 14); } \\
\text { Alto risco (escore de } 15 \text { a } \\
\text { 19); } \\
\text { Altíssimo risco (escore } \geq 20 \text { ). } \\
\text { (13) }\end{array}$ & $\begin{array}{l}\text { Quanto menor o escore, } \\
\text { maior risco de UP: } \\
\text { Risco muito alto (escores } \leq 9 \text { ); } \\
\text { Risco alto (escores de } 15 \text { a } 18 \\
\text { pontos); } \\
\text { Sem risco (escores } \geq 19 \text { ). } \\
\text { (14) }\end{array}$ \\
\hline
\end{tabular}

Fonte: elaborado pelas autoras com base em dados bibliográficos, Salvador (2012).

A Escala de Norton avalia cinco parâmetros para grau de risco: condição física, nível de consciência, atividade, mobilidade e incontinência. Cada parâmetro foi pontuado com valores de 1 a 4, chegando a um total de 20 pontos. Sendo assim, quanto menor for o somatório final, maior será o risco para o desenvolvimento de UP, estando mais susceptível em pacientes com pontuação inferior a 12 pontos. Foi a pioneira das escalas, sendo formulada em $1962 .^{(6)}$

A Escala de Waterlow se baseou na Escala de Norton, porém abrange mais classificações, utilizando sete parâmetros principais para identificar os fatores causais, oferecendo um método de avaliação de risco e grau da lesão. Esses parâmetros são: relação peso/altura (IMC), avaliação visual da pele em áreas de risco, sexo/idade, continência, mobilidade, apetite e medicações. Além deles, existem mais quatro outros itens que pontuam os fatores de risco especiais, sendo eles subnutrição do tecido celular, déficit neurológico, tempo de cirurgia acima de duas horas e trauma abaixo da medula lombar. O escore indica o risco de desenvolver ou não a UP, sendo que, quanto mais alto o escore, maior o risco. A estratificação ocorre em três grupos: em risco (escore de 10 a 14), alto risco (escore de 15 a 19) e altíssimo risco (escore $\geq 20$ ). Nessa escala, quanto maior a idade do paciente, maior será a pontuação. ${ }^{(13)}$

A Escala de Braden integra seis subescalas: percepção sensorial, atividade, mobilidade, umidade, nutrição, fricção ou cisalhamento. Essas escalas são pontuadas de um a 
quatro, exceto fricção ou cisalhamento, cuja medida varia de um a três. O escore total pode variar de 6 a 23 pontos, sendo os pacientes classificados da seguinte forma: risco muito alto (escores $\leq 9$ ), risco alto (escores de 15 a 18 pontos) e sem risco (escores $\geq 19$ ). ${ }^{(3,14)}$

Em um estudo realizado por Araújo, Araújo, Caetano, ${ }^{(6)}$ as Escalas de Norton e Braden foram denominadas como negativas e a de Waterlow como positiva, ou seja, quanto menor os pontos, maior a vulnerabilidade do paciente em desenvolver UP, conforme as escalas de Norton e Braden, e diminuição do risco de acordo com Waterlow. A Escala de Waterlow, em virtude dessa elevação de pontos, evidenciou mais casos, estatisticamente significantes, de desenvolvimento de UP do que os dois outros instrumentos. Nos serviços de saúde que apenas utilizam Norton e Braden talvez ocorra uma subestimação de casos de pacientes vulneráveis para o desenvolvimento dessas lesões. Para esses autores, a Escala de Waterlow é um instrumento de alta sensibilidade $(50,6 \%)$ e especificidade $(60,1 \%)$.

Em contrapartida, Menegon et al. ${ }^{(15)}$ realizou um estudo por meio da aplicação da Escala de Braden em uma determinada instituição demonstrando que a mesma atingiu $94 \%$ de sensibilidade, $89 \%$ de especificidade, $88 \%$ de validade preditiva para teste positivo e $94 \%$ para teste negativo. Esses dados conferiram que o uso da Escala de Braden permite a mensuração objetiva do risco de desenvolver UP apresentado pelo paciente e fornece subsídios para a elaboração do diagnóstico de enfermagem de risco da integridade da pele, o que pode reduzir a incidência dessas lesões em até $50 \%$ dos casos.

Ficou evidente nos estudos revisados que a não percepção sensorial torna os pacientes incapazes de comunicar o desconforto, tornando-se mais vulneráveis para o desenvolvimento de UP. Das escalas analisadas a única que avalia a percepção sensorial é a de Braden, justificando a especificidade dessa escala e a sua maior utilização comparada com as demais, sendo o instrumento de escolha para a maior parte dos estudos pesquisados..$^{(8,9,16,17)}$

Diferente dos autores acima citados, Rocha e Barros ${ }^{(13)}$ abordam que a avaliação da pele apresenta extrema importância na caracterização do risco de desenvolver UP, demonstrado através de estudo feito com 44 pacientes, evidenciando que $96 \%$ dessa amostra desenvolveram UP por apresentarem pele muito fina, seca ou com edema. A única escala estudada que avalia a pele é a de Waterlow, que possui uma subescala denominada avaliação visual da pele em áreas de risco, tornando-se mais específica na identificação do risco de desenvolver UP.

Nota-se nos estudos revisados entre os autores que há divergência na frequência da aplicação da Escala de Braden. Apesar de Fernandes e Caliri ${ }^{(16)}$ recomendarem sua aplicação 
na admissão e a cada 48 horas, Bavaresco, Medeiros, Lucena ${ }^{(7)}$ realizaram um estudo com 45 pacientes nos quais houve o preenchimento diário da Escala de Braden e apenas cinco desenvolveram UP, comparado a 29 casos em que não houve a aplicação diária da escala de Braden e 14 deles desenvolveram. Isso demonstra que não há consenso quanto à frequência de aplicação. Segundo Serpa et al, ${ }^{(3)}$ em recente revisão sobre a prevenção de UP, desenvolvida pela National Pressure Ulcer Advisory Panel (NPUAP), o organismo recomenda que os protocolos institucionais determinem a realização da avaliação de risco e reavaliações de acordo com as características das unidades clínicas onde o paciente está sendo atendido.

Em comparação com as demais escalas estudadas, segundo Bravin e Ribeiro, ${ }^{(18)}$ a desvantagem da Escala de Norton é que ela não contempla alguns fatores de risco importantes para o desenvolvimento de UP, como por exemplo, idade do paciente, fricção, cisalhamento e as condições da pele. Além disso, por não possuir especificidade nas pontuações, podem ocorrer divergências de opiniões dos profissionais, tornando-se menos eficaz na prevenção de UP.

\section{O papel do enfermeiro na aplicação das escalas}

A atuação da equipe de enfermagem em uma unidade hospitalar visa o atendimento do paciente crítico, incluindo o diagnóstico situacional, intervenções e avaliação dos cuidados específicos de enfermagem, a partir de uma visão humanista voltada para a qualidade de vida. O reconhecimento dos indivíduos em risco de desenvolver UP não depende somente da habilidade clínica do profissional, mas do uso de um instrumento de medida, como as escalas de avaliação, que apresentam adequados índices de validade preditiva, aplicabilidade e especificidade. $^{(8)}$

Segundo Rogenski e Kurcgant ${ }^{(14)}$ e Silva et al. ${ }^{(8)}$ recomenda-se o uso da escala para auxiliar o profissional a identificar os pacientes de risco já na admissão, norteando a adoção das medidas preventivas no cuidado, porém, para a efetividade da escala é imprescindível que haja o máximo de concordância entre diferentes profissionais em relação à pontuação das subescalas e no total das mesmas. A diferença de conhecimento entre os profissionais que realizam essa prática poderá ocasionar interpretações variadas tornando-a subjetiva e perdendo assim sua validade.

Torna-se necessário capacitar os profissionais de enfermagem para a utilização de escalas que predizem o risco de desenvolvimento de UP, pois equivalem a uma sistematização 
do atendimento ao paciente, incluindo diagnóstico em termo dessas lesões, intervenções de enfermagem através das recomendações e avaliação dos resultados do cuidado implementado. ${ }^{(4)}$

Além de considerar a gravidade do estado clínico do paciente, é importante um dimensionamento da equipe de enfermagem conforme a demanda de cuidados específicos, pois o excesso da carga de trabalho é um fator diretamente relacionado à abertura dessas lesões, interferindo na qualidade da assistência prestada ao paciente, na qualidade de vida dos profissionais e nos custos hospitalares. Acrescido a isso, existe a valorização e prioridade dos cuidados intensivos para estabilização hemodinâmica do paciente comparado a implementação de medidas preventivas para UP. ${ }^{(2)}$

\section{CONSIDERAÇÕES FINAIS}

A utilização das escalas preditivas é fundamental para a atuação do enfermeiro, pois é um preditor importante na identificação da UP, reforçando a necessidade de examinar a pele do paciente, instalar insumos preventivos e monitorá-lo com instrumentos de poder preditivo, positivo e negativo eficazes. Além disso, elas apontam pontos vulneráveis, reforçam a necessidade de avaliação constante e estimulam a prevenção. Todavia, é necessário além da destreza, que o enfermeiro adote instrumentos mais viáveis e eficazes para o seu trabalho.

$\mathrm{Na}$ avaliação clínica do paciente, devem ser levados em consideração os diversos fatores que predispõem à formação de UP, pois essa lesão não ocorre apenas por um determinado fator de risco, mas pela relação dos diversos fatores predisponentes, o que reforça a importância da aplicação de um instrumento que avalie estes riscos.

Por meio deste estudo, pôde-se concluir que a escala com maior preditividade e sensibilidade segundo a maioria dos autores foi a de Braden, pois a sua utilização na prática clínica é bastante útil para predizer o desenvolvimento de UP ou sua recidiva, permitindo conhecer o risco individual de cada paciente e implementar, precocemente, ações de enfermagem preventiva e condizentes com esse risco. 


\section{REFERÊNCIAS}

1. Wada A, Neto NT, Ferreira MC. Úlcera por pressão. Rev. Med. São Paulo. 2010 [acesso em: 30 Mar 2012]; 3(89):170-7. Disponível em: http://www.revistademedicina.org.br/ant/893/14-ulceras\%20pressao.pdf

2. Cremasco MF et al. Úlcera por pressão: risco e gravidade do paciente e carga de trabalho de enfermagem. Rev. paul. enferm. São Paulo, 2009 [acesso em: 30 Mar 2012];22(1):897902. Disponível em: http://www.scielo.br/pdf/ape/v22nspe/11.pdf

3. Serpa LF et al. Validade preditiva da Escala de Braden para o risco de desenvolvimento de úlcera por pressão em pacientes críticos. Rev. latinoam. Enferm. São Paulo. 2011 [acesso em: 30 Mar 2012];1(19) Disponível em: http://www.scielo.br/pdf/rlae/v19n1/pt_08.pdf

4. Sousa CAC, Santos I, Silva LD. Aplicando recomendações da escala de Braden e prevenindo úlceras por pressão: evidências do cuidar em enfermagem. Rev. bras. enferm. Brasília, 2006 [acesso em: 20 Ago 2012];59(3). Disponível em: http://bases.bireme.br/cgibin/wxislind.exe/iah/online/?IsisScript=iah/iah.xis\&src=google\&base=LILACS\&lang=p\&nex tAction=lnk\&exprSearch=480908\&indexSearch=ID

5. Gomes FS et al. Avaliação de Risco para Úlcera por Pressão em Pacientes Críticos. Rev. Esc. Enferm. USP, São Paulo. 2011 [acesso em: 31 Mar 2012];45(2):313-18. Disponível em: http://www.scielo.br/pdf/reeusp/v45n2/v45n2a01.pdf

6. Araújo TM, Araújo MFM, Caetano JA. Comparação de escalas de avaliação de risco para úlcera por pressão em pacientes em estado crítico. Rev. paul. enferm. São Paulo. 2011 [acesso em: 15 Ago 2012];24(5):695-700. Disponível em:

http://www.scielo.br/scielo.php?script=sci_arttext\&pid=S0103-

21002011000500016\&lng=en\&nrm=iso\&tlng=pt

7. Bavaresco T, Medeiros RH, Lucena AF. Implantação da Escala de Braden em uma Unidade de Terapia Intensiva de um Hospital Universitário. Rev. gaúch. enferm. Porto Alegre. 2011[acesso em: 27 Mar 2012];32(4):703-10. Disponível em: http://seer.ufrgs.br/RevistaGauchadeEnfermagem/article/view/17469/14445

8. Silva EW et al. Aplicabilidade do protocolo de prevenção de úlcera de pressão em Unidade de Terapia Intensiva. Rev. bras. ter. intensiva. Recife. 2010 [acesso em: 30 Abr 2012];2(22): 175-185. Disponível em: http://www.scielo.br/pdf/rbti/v22n2/a12v22n2.pdf

9. Gomes FSL et al. Fatores associados à úlcera por pressão em pacientes internados nos centro de terapia intensiva de adultos. Rev. Esc. Enferm. USP, 2010 [acesso em: 20 Ago 2012];44(4):1070-6. Disponível em: http://www.scielo.br/pdf/reeusp/v44n4/31.pdf .

10. Mendes KDS, Silveira RCCP, Galvão CM. Revisão integrativa: método de pesquisa para a incorporação de evidências na saúde e na enfermagem. Texto \& contexto enferm. 2008 [acesso em: 17 Maio 2012];17(4):758-764. Disponível em: http://redalyc.uaemex.mx/src/inicio/ArtPdfRed.jsp?iCve=71411240017 
11. Andrade MM. Como preparar trabalhos para cursos de pós graduação: noções práticas. 3 ed. São Paulo: Atlas, 2002. Cap. 3, p. 131-141.

12. Blanes L et al. Avaliação clínica e epidemiológica das úlceras por pressão em pacientes internados no Hospital São Paulo. AMB rev. Assoc. Med. Bras. São Paulo. 2004 [acesso em: 30 mar. 2012]; 50(2):182-7. Disponível em: http://www.scielo.br/pdf/ramb/v50n2/20781.pdf

13. Rocha ABL, Barros SM. O. Avaliação de risco de úlcera por pressão: propriedades de medida da versão em português da Escala de Waterlow. Rev. paul. enferm. São Paulo, 2007 [acesso em: 30 mar. 2012];2(20). Disponível em: http://www.scielo.br/scielo.php?pid=S0103$21002007000200006 \&$ script=sci_arttext

14. Rogenski NMR, Kurcgant P. Avaliação da concordância da aplicação da Escala de Braden interobservadores. Rev. paul. enferm. São Paulo. 2012 [acesso em: 20 Ago 2012]; 25(1):24-28. Disponível em: http://bases.bireme.br/cgibin/wxislind.exe/iah/online/?IsisScript=iah/iah.xis\&src=google \&base=LILACS\&lang=p\&nex tAction=lnk\&exprSearch=617975\&indexSearch=ID.

15. Menegon DB et al. Implantação do protocolo assistencial de prevenção e tratamento de úlcera de pressão no Hospital de Clínicas de Porto Alegre. Rev. HCPA \& Fac. Med. Univ. Fed. Rio Gd. do Sul. Porto Alegre. 2007 [acesso em: 21 Ago 2012];27(7). Disponível em: http://seer.ufrgs.br/hcpa/article/view/2031.

16. Fernandes LM, Caliri MHL. Uso da Escala de Braden e de Glasgow para identificação do risco para úlceras de pressão em pacientes internados em Centros de Terapia Intensiva. Rev. latinoam enferm. São Paulo, Nov/Dez 2008. [acesso em: 20 Ago 2012]

Disponível em: http://www.scielo.br/pdf/rlae/v16n6/pt_06.

17. Sousa CAC, Santos I, Silva LD. Apropriação de concepções de Neuman e Braden na prevenção de úlceras de pressão. Rev. enferm. UERJ, Rio de Janeiro. 2004 [acesso em: 20 Ago 2012]: 280-5. Disponível em: http://bases.bireme.br/cgibin/wxislind.exe/iah/online/?IsisScript=iah/iah.xis\&src=google\&base=LILACS\&lang=p\&nex tAction=lnk\&exprSearch=397731\&indexSearch=ID.

18. Bravin M, Ribeiro A G. Proposição de estratégias para prevenção de úlceras de pressão em usuários acamados nos serviços de saúde. São Paulo. 2005 [acesso em: 2 Abr 2012]; 37(4). Disponível em: http://189.75.118.67/CBCENF/sistemainscricoes/arquivosTrabalhos/Proposicao\%20de\%20est rategias.pdf 